\title{
MyD88-BLT2-dependent cascade contributes to LPS-induced interleukin-6 production in mouse macrophage
}

\author{
A-Jin Lee ${ }^{1}$, Kyung-Jin $\mathrm{Cho}^{1}$ and Jae-Hong Kim \\ Endotoxic responses to bacterial lipopolysaccharide (LPS) are triggered by Toll-like receptor 4 (TLR4) and involve the production \\ of inflammatory mediators, including interleukin-6 (IL-6), by macrophages. The detailed mechanism of IL-6 production by \\ macrophages in response to LPS has remained unclear, however. We now show that LPS induces IL-6 synthesis in mouse \\ peritoneal macrophages via the leukotriene $B_{4}$ receptor BLT2. Our results suggest that TLR4-MyD88 signaling functions \\ upstream of BLT2 and that the generation of reactive oxygen species (ROS) by NADPH oxidase 1 (Nox1) and consequent \\ activation of the transcription factor nuclear factor (NF)-кB function downstream of BLT2 in this response. These results suggest \\ that a TLR4-MyD88-BLT2-Nox1-ROS-NF-кB pathway contributes to the synthesis of IL-6 in LPS-stimulated mouse \\ macrophages.
}

Experimental \& Molecular Medicine (2015) 47, e156; doi:10.1038/emm.2015.8; published online 3 April 2015

\section{INTRODUCTION}

Sepsis, a chronic systemic inflammatory response syndrome, is a leading cause of death in critically ill patients. ${ }^{1}$ Despite significant advances in critical care, there is still no Food and Drug Administration-approved pharmacological therapy applicable to patients indicating the urgent need to further elucidate the molecular pathways or new players leading to the pathology of endotoxic shock. The most lethal form of sepsis, endotoxic shock, is caused by lipopolysaccharide (LPS) released from the surface of replicating Gram-negative bacteria into the circulation, where it is recognized by various immune cells, including macrophages. ${ }^{2}$ The interaction of LPS with macrophages triggers the production of various pro-inflammatory cytokines, including tumor necrosis factor- $\alpha$, interleukin (IL)$1 \beta$ and IL-6, contributing to the pathogenesis of endotoxic shock. ${ }^{3,4}$ The production of IL-6 is a hallmark of endotoxic shock, with high levels of this cytokine in affected individuals being associated with mortality. Despite the central role of IL-6 in endotoxin-induced inflammatory pathogenesis, however, the signaling events responsible for triggering IL- 6 production by LPS in macrophages have still remained unclear.

Toll-like receptor 4 (TLR4) is a pathogen-recognition receptor that has a key role in the recognition of LPS and in eliciting the subsequent inflammatory response. ${ }^{5}$ The interaction of LPS with TLR4 triggers the recruitment of the cytoplasmic adaptor protein MyD88 (myeloid differentiation factor 88) and the consequent activation of nuclear factor $(\mathrm{NF})-\kappa \mathrm{B}$, which induces the expression of pro-inflammatory cytokine genes, such as IL-6. ${ }^{6}$ This TLR4-MyD88 pathway has been thought to have an important role in LPS-induced IL-6 synthesis. ${ }^{7,8}$ Also, previous studies have shown that mice deficient in cytosolic phospholipase $\mathrm{A}_{2}\left(\mathrm{CPLA}_{2}\right)$ manifest reduced synthesis of IL- 6 during experimental sepsis and that LPS-induced IL-6 production in macrophages is regulated through activation of $\mathrm{CPLA}_{2}$ mediated by $\mathrm{p} 38$ mitogenactivated protein kinase (MAPK), ${ }^{9,10}$ suggesting that $\mathrm{CPLA}_{2}$ signaling also contributes to the LPS-induced production of IL-6 in macrophages. The downstream components of this $\mathrm{CPLA}_{2}$ signaling pathway have remained unclear, however.

We have now found that BLT2, a leukotriene $B_{4}$ receptor-2, functions downstream of $\mathrm{CPLA}_{2}$ in the induction of IL-6 synthesis by LPS in mouse macrophages and that BLT2 expression is upregulated by LPS in a MyD88-dependent manner. The levels of the BLT2 ligands leukotriene $\mathrm{B}_{4}$ $\left(\mathrm{LTB}_{4}\right)$ and $12(S)$-hydroxyeicosatetraenoic acid (12(S)-HETE) as well as of their biosynthetic enzymes 5-lipoxygenase (5-LO) and 12-lipoxygenase (12-LO) are all increased by LPS in macrophages. In addition, the generation of reactive oxygen

\footnotetext{
College of Life Sciences and Biotechnology, Korea University, Seoul, Korea

${ }^{1}$ These authors contributed equally to this work.

Correspondence: Professor J-H Kim, College of Life Sciences and Biotechnology, Korea University, 5-1 Anam-dong, Sungbuk-gu, Seoul 136-701, Korea. E-mail: jhongkim@korea.ac.kr

Received 23 September 2014; revised 15 December 2014; accepted 18 December 2014
} 
species (ROS) by NADPH oxidase 1 (Nox1) was found to occur downstream of BLT2, contributing to IL-6 production via activation of NF- $\mathrm{KB}$. Together, our observations suggest that a TLR4-MyD88-CPLA $2-B L T 2-N o x 1-R O S-N F-\kappa B$ pathway greatly contributes to the synthesis of IL-6 in LPSstimulated mouse macrophages. Targeting this pathway might prove beneficial for endotoxin-induced lung inflammation therapy.

\section{MATERIALS AND METHODS}

\section{Reagents}

LPS (Escherichia coli serotype O55:B5), thioglycollate medium, dimethyl sulfoxide, diphenyleneiodonium (DPI) and $\mathrm{N}$-acetylcysteine (NAC) were obtained from Sigma (St Louis, MO, USA). 2',7'Dichlorofluorescein diacetate was from Molecular Probes (Eugene, OR, USA); baicalein, $\mathrm{AACOCF}_{3}, \mathrm{AA} 861$ and Bay11-7082 were from Calbiochem (La Jolla, CA, USA); and SB203580 was from Calbiochem (San Diego, CA, USA).

\section{Cell culture and isolation of mouse peritoneal macrophages} Raw 264.7 mouse macrophages (American Type Culture Collection, Manassas, VA, USA) were maintained under a humidified atmosphere of $5 \% \mathrm{CO}_{2}$ at $37^{\circ} \mathrm{C}$ in RPMI 1640 medium supplemented with $10 \%$ fetal bovine serum, $0.1 \mathrm{~mm}$ nonessential amino acids and antibioticantimycotic solution (Invitrogen, Grand Island, NY, USA). Mouse peritoneal macrophages were isolated from peritoneal fluid harvested 4 days after intraperitoneal injection of the animals with $2 \mathrm{ml}$ of $10 \%$ thioglycollate medium. Cells isolated from the fluid by centrifugation at 1000 r.p.m. for $5 \mathrm{~min}$ at room temperature were seeded in complete RPMI 1640 medium and allowed to attach to culture plates for $4 \mathrm{~h}$, after which the non-adherent cells were removed by vigorous washing with phosphate-buffered saline. The adherent cells were stained with fluoresecin isothiocyanate-conjugated rat monoclonal antibodies to mouse CD11b or an immunoglobulin G2a isotype control antibody (BD Pharm Lyse; BD Biosciences, San Diego, CA, USA) and then analyzed by flow cytometry (FACSCalibur instrument; Becton Dickinson, Frankin Lakes, NJ, USA). The purity of the peritoneal macrophage preparations was usually $>92 \%$. The cells were allowed to attach to the bottom of culture plates for $24 \mathrm{~h}$ and then incubated in medium containing $0.5 \%$ fetal bovine serum for $6 \mathrm{~h}$ before exposure to test agents in the same medium for the indicated times.

\section{Semiquantitative and quantitative reverse transcriptase PCR analysis}

Total RNA was extracted from cells or homogenized lung tissue with the use of Easy Blue (Intron, Sungnam, Korea), and portions $(2 \mu \mathrm{g})$ of the RNA were subjected to reverse transcriptase with M-MLV reverse transcriptase (Beams Bio, Kyunggi, Korea) followed by semiquantitative PCR analysis with a PCR PreMix Kit (Intron) under conditions found to be optimal for linear amplification of glyceraldehyde 3-phosphate dehydrogenase (GAPDH) cDNA. The primers (forward and reverse, respectively) included 5'-ACTGGCCTGAGC AACTAGGA-3' and 5'-CGTGCCACTACCTGTAGCAA-3' for MyD88, 5'-AATGCCCAGGATCGAGGT- ${ }^{\prime}$ and 5'-GATGGAAGCAAA GGGAGTGA-3' for Nox1, and 5'-CACGGCCTTCCCTACTTCA-3' and $5^{\prime}$-TCTGGCTTTGTCTTTCTTGTTATC- $3^{\prime}$ for IL-6. The primer for BLT2 was described previously. ${ }^{11,12}$ The specificity of all primers was confirmed by sequencing of the PCR products. Real-time PCR analysis was performed with the use of LightCycler 480 SYBR Green I
Master (Roche Diagnostics, Mannheim, Germany). All data were normalized by the abundance of GAPDH.

\section{RNA interference}

Cells were transfected with MyD88 or BLT2 (Bioneer, Daejeon, Korea) or with Nox1 small interfering RNAs (siRNAs; Invitrogen) with the use a MP-100 Microporator (Digital Bio, Seoul, Korea). In brief, cells $\left(1 \times 10^{6}\right)$ in $100 \mu \mathrm{l}$ of resuspension buffer containing test or control (scrambled) siRNAs (50 nM) were subjected to electroporation with two pulses of $1500 \mathrm{~V}$ for $20 \mathrm{~ms}$ (peritoneal macrophages) or one pulse of $1500 \mathrm{~V}$ for $40 \mathrm{~ms}$ (Raw 264.7 cells). All cells were then cultured in complete medium but without antibiotics for $24 \mathrm{~h}$ before analysis or experiments.

\section{Forced expression of MyD88 or BLT2}

Cells $\left(1 \times 10^{6}\right)$ were transiently transfected with $2 \mu \mathrm{g}$ of expression vectors for human MyD88 (pCMV-Flag-MyD88, kindly provided by T Renno) ${ }^{13}$ or with the corresponding empty vector (pCMV-Flag), with the use of an MP-100 Microporator (Digital Bio).

\section{Measurement of IL-6, $\mathrm{LTB}_{4}$ and 12(S)-HETE}

Conditioned medium from macrophage incubations was immediately frozen and lyophilized. The amounts of IL-6 (BD Biosciences) and of $\mathrm{LTB}_{4}$ and 12(S)-HETE (Assay Designs, Ann Arbor, MI, USA) were quantified with the use of enzyme-linked immunosorbent assay kits.

\section{Preparation of cell lysates and immunoblot analysis}

The phosphate-buffered saline-washed cells were lysed into a lysis buffer (20 mм Tris- $\mathrm{HCl}$ (pH 7.5), $150 \mathrm{~mm} \mathrm{NaCl}, 0.5 \%$ Nonidet P-40, $5 \mathrm{~mm}$ EDTA, $1 \%$ Triton X-100, $100 \mathrm{~mm}$ phenylmethylsulfonyl fluoride, $1 \mathrm{~mm}$ sodium orthovanadate, leupeptin $\left(2 \mu \mathrm{g} \mathrm{ml}{ }^{-1}\right)$, aproti$\left.\operatorname{nin}\left(2 \mu \mathrm{g} \mathrm{ml}{ }^{-1}\right)\right)$ at $4{ }^{\circ} \mathrm{C}$ and heated at $95^{\circ} \mathrm{C}$ for $5 \mathrm{~min}$. The lysates were then subjected to sodium dodecyl sulfate-polyacrylamide gel electrophoresis, and the separated proteins were transferred electrophoretically to a polyvinylidene difluoride membrane for $90 \mathrm{~min}$ at $100 \mathrm{~V}$. The membrane was exposed for $1 \mathrm{~h}$ to Tris-buffered saline containing $0.05 \%$ Tween 20 and 5\% dried nonfat milk before incubation overnight at $4{ }^{\circ} \mathrm{C}$ with primary antibodies at a dilution of 1:2000 (or 1:4000 in the case of those to $\beta$-actin) in Tris-buffered saline containing $0.05 \%$ Tween 20 . The membrane was then incubated for $2 \mathrm{~h}$ at room temperature with horseradish peroxidise-conjugated secondary antibodies before detection of immune complexes with the use of an enhanced chemiluminescence kit (Amersham Biosciences, Little Chalfont, UK). Antibodies to cPLA, $5-\mathrm{LO}$ or 12-LO were from Santa Cruz Biotechnology (Santa Cruz, CA, USA), and antibodies to $p 38$, phospho-p38, IкB $\alpha$, to phospho-I $\mathrm{KB} \alpha$ or $\beta$-actin (loading control) were obtained from Cell Signaling Technology (Danvers, MA, USA). Size estimates for proteins were obtained using molecular weight standards from Thermo scientific (Rockford, IL, USA).

\section{Measurement of ROS}

Cells were incubated for final $20 \mathrm{~min}$ with the ROS-sensitive fluorophore 2',7'-dichlorofluorescein diacetate $(10 \mu \mathrm{M})$, washed with phosphate-buffered saline and immediately analyzed for intracellular ROS levels by measurement of DCF fluorescence with a flow cytometer (FACSCalibur) 


\section{Statistical analysis}

Data are presented as means \pm SD and were analyzed by one-way analysis of variance or Student's $t$-test for comparisons among multiple or between two groups, respectively. A $P$-value of $<0.05$ was considered statistically significant.

\section{RESULTS}

Role of BLT2 in LPS-induced IL-6 synthesis in macrophages Consistent with previous observations, ${ }^{10}$ the expression of IL- 6 at both mRNA and protein levels in mouse peritoneal macrophages was increased by LPS in a time-dependent manner (Figures 1a and b). The abundance of BLT2 mRNA was also markedly increased in these cells by LPS (Figure 1a). We next examined whether BLT2 contributes to LPS-induced IL-6 synthesis. Depletion of BLT2 with a specific siRNA (Figures 1c and d) greatly attenuated LPS-induced IL-6 expression. Together, these data suggested that BLT2 has a critical role in LPS-induced IL-6 synthesis in mouse macrophages. Interestingly, we found that BLT2 inhibition had no effect on LPS-induced expression of tumor necrosis factor- $\alpha$ or IL- $1 \beta$ (data not shown), suggesting that LPSinduced IL-6 synthesis is preferentially regulated through a BLT2-dependent mechanism. a

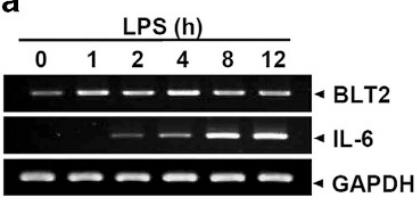

c

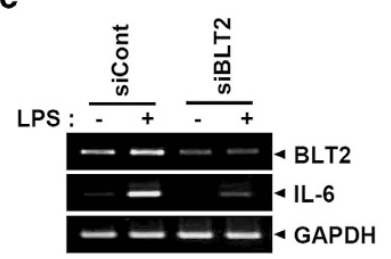

b

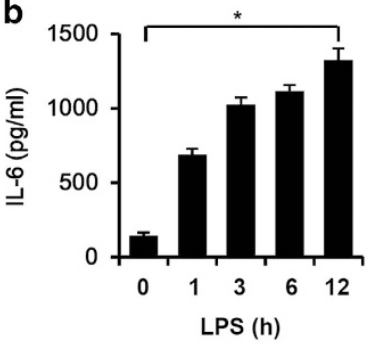

d

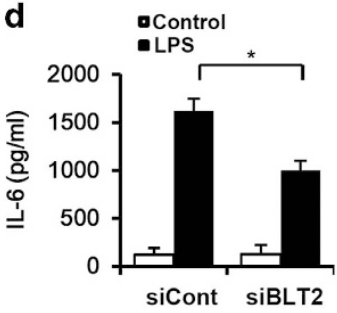

Figure 1 LPS-induced IL- 6 synthesis is dependent on BLT2 in mouse macrophages. (a and $\mathbf{b}$ ) Peritoneal macrophages were stimulated with LPS $\left(100 \mathrm{ng} \mathrm{ml}^{-1}\right)$ for the indicated times, after which total RNA was isolated and subjected to RT-PCR analysis (a) and IL-6 released into the culture medium was assayed (b). (c) Peritoneal macrophages were transfected with control (siCont) or BLT2 (siBLT2) siRNAs. After $24 \mathrm{~h}$, the cells were incubated in the absence or presence of LPS for $4 \mathrm{~h}$, and total RNA was then isolated and subjected to RT-PCR analysis. (d) Peritoneal macrophages were transfected with control or BLT2 siRNAs, incubated for $24 \mathrm{~h}$ and then stimulated with LPS for $12 \mathrm{~h}$, after which IL-6 released into the culture medium was assayed. All quantitative data are means \pm s.d. from three independent experiments. ${ }^{*} P<0.05$.

\section{Upregulation of BLT2 ligands in LPS-stimulated} macrophages

Ligands for BLT2 include eicosanoids such as $\mathrm{LTB}_{4}, 12(S)$ HETE and 12(S)-hydroxyheptadeca-5Z,8E,10E-trienoic acid. ${ }^{14}$ The synthesis of $\mathrm{LTB}_{4}$ and $12(\mathrm{~S})$-HETE from arachidonic acid is catalyzed by $5-\mathrm{LO}$ and $12-\mathrm{LO}$, respectively. ${ }^{15}$ To examine whether these BLT2 ligands contribute to LPS-induced IL-6 synthesis in macrophages, we first determined the amounts of $\mathrm{LTB}_{4}$ and 12(S)-HETE. They were increased in a timedependent manner following LPS stimulation in mouse peritoneal macrophages (Figure 2a). These effects of LPS were attenuated by prior exposure of the cells to the 5-LO inhibitor AA861 or the 12-LO inhibitor baicalein, respectively (Figure 2b). In addition, the $\mathrm{CPLA}_{2}$ inhibitor $\mathrm{AACOCF}_{3}$ suppressed the LPS-induced production of both these BLT2 ligands (Figure 2b). LPS-induced IL-6 expression at the mRNA and protein levels was also significantly attenuated by the inhibitors of 5-LO, 12- $\mathrm{LO}$ and $\mathrm{CPLA}_{2}$ in both peritoneal macrophages (Figures 2c and d) and Raw 264.7 cells (data not shown). Furthermore, the amounts of 5-LO and 12-LO were markedly increased in response to LPS stimulation in Raw 264.7 cells (Figure 2e). In addition, activation of $\mathrm{CPLA}_{2}$, as revealed by its phosphorylation at $\mathrm{Ser}^{505}$, was evident at $15 \mathrm{~min}$ and maximal at $30 \mathrm{~min}$, whereas the total abundance of $\mathrm{CPLA}_{2}$ remained unchanged (Figure 2f). Together, these results suggested that LPS-induced generation of the BLT2 ligands $\mathrm{LTB}_{4}$ and 12(S)-HETE via the consecutive action of $\mathrm{CPLA}_{2}$ and that either 5-LO or 12-LO is required for the upregulation of IL-6 synthesis by this bacterial component.

\section{MyD88 functions upstream of $\mathrm{LTB}_{4} / 12$ (S)-HETE-BLT2 in} LPS-induced IL-6 synthesis

Previous studies indicated that TLR4 and the cytoplasmic adaptor protein MyD88 mediate LPS-induced IL-6 synthesis. ${ }^{7}$ We therefore next tested whether the upregulation of BLT2 and 5-LO/12-LO in mouse macrophages is dependent on MyD88. Depletion of MyD88 by siRNA transfection resulted in marked attenuation of the LPS-induced increase in the abundance of BLT2 mRNA in both peritoneal macrophages (Figure 3a) and Raw 264.7 cells (Figure 3b). Depletion of MyD88 also inhibited the LPS-induced increases in the amounts of 5-LO and 12-LO (Figure 3c) as well as in the level of $\mathrm{cPLA}_{2}$ phosphorylation (Figure 3d) in Raw 264.7 cells. Consistent with these results, the LPS-induced generation of $\mathrm{LTB}_{4}$ and 12(S)-HETE in mouse peritoneal macrophages was significantly inhibited by transfection with MyD88 siRNA (Figures 3e and f). Furthermore, knockdown of MyD88 suppressed the LPS-induced expression of IL-6 at both the mRNA and protein levels (Figures $3 \mathrm{~g}$ and $\mathrm{h}$ ). We then examined the effects of transient transfection of cells with a MyD88 expression plasmid. ${ }^{13}$ Overexpression of MyD88 alone increased the levels of 5-LO and $12-\mathrm{LO}$ as well as the extent of $\mathrm{CPLA}_{2}$ phosphorylation in Raw 264.7 cells (Figure 3i). In addition, the production of $\mathrm{LTB}_{4}$ and 12(S)-HETE was significantly increased by forced expression of MyD88 (Figures $3 \mathrm{j}$ and $\mathrm{k}$ ). Finally, the amounts of BLT2 mRNA as well as IL-6 mRNA and protein were all 
a

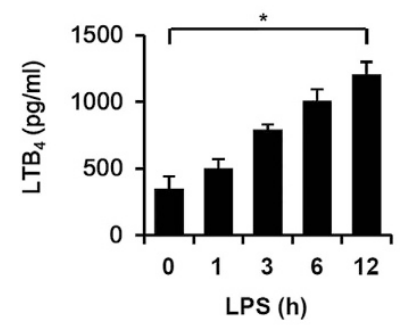

C

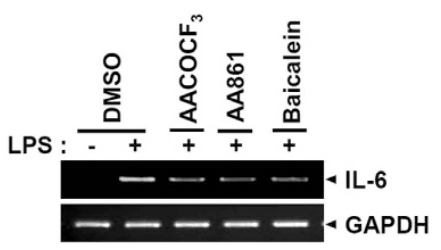

b
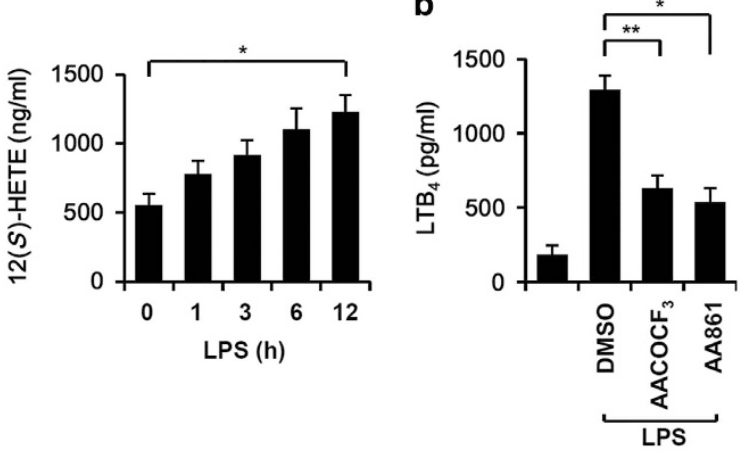

e

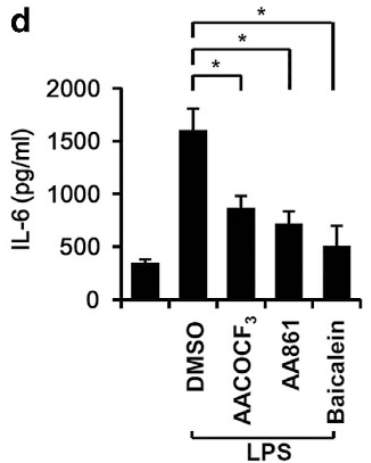

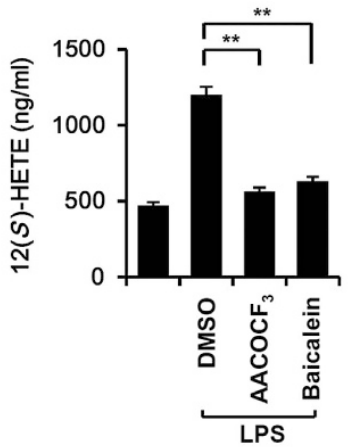

$\mathbf{f}$

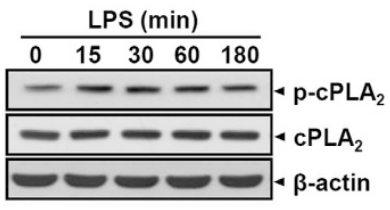

Figure 2 LPS-induced IL-6 synthesis is dependent on 5-LO and 12-LO in mouse macrophages. (a) Peritoneal macrophages were stimulated with LPS $\left(100 \mathrm{ng} \mathrm{ml}^{-1}\right)$ for the indicated times, after which $\mathrm{LTB}_{4}$ and $12(\mathrm{~S})$-HETE released into the culture medium were assayed. (b) Peritoneal macrophages were incubated first for $30 \mathrm{~min}$ with $\mathrm{AACOCF}_{3}(20 \mu \mathrm{m})$, AA861 (10 $\left.\mu \mathrm{m}\right)$, baicalein $(20 \mu \mathrm{m})$ or DMSO and then for $12 \mathrm{~h}$ in the absence or presence of LPS, after which LTB 4 and 12(S)-HETE released into the culture medium were assayed. (c) Peritoneal macrophages were incubated first for $30 \mathrm{~min}$ with inhibitors as in panel (b) and then for $4 \mathrm{~h}$ in the absence or presence of LPS, after which total RNA was isolated and subjected to semiquantitative RT-PCR analysis. (d) The culture supernatants of peritoneal macrophages treated as in panel (b) were assayed for IL-6. (e and f) Raw 264.7 cells were stimulated with LPS for the indicated times, after which cell lysates were subjected to immunoblot analysis. All quantitative data are means \pm s.d. from three independent experiments. ${ }^{*} P<0.05,{ }^{*} P<0.01$.

increased by MyD88 overexpression in Raw264.7 cells or primary peritoneal macrophages (Figures 31 and $\mathrm{m}$ ). Together, these results suggested that a $\mathrm{CPLA}_{2}-\mathrm{LTB}_{4} / 12(\mathrm{~S})$-HETE-BLT2 signaling cascade potentially is activated via MyD88 and contributes to LPS-induced IL-6 synthesis in macrophages.

Nox1-ROS signaling functions downstream of BLT2 in LPSinduced IL- 6 synthesis

We previously showed that NADPH oxidase (Nox)-derived ROS function as downstream mediators of BLT2 in various cell types. ${ }^{16-18}$ ROS generation has also been found to be important for LPS signaling in macrophages, and LPS-induced ROS generation in these cells is attenuated by DPI, an inhibitor of flavoenzymes, including Nox. ${ }^{19-21}$ We therefore investigated whether BLT2-dependent, Nox-mediated ROS generation might contribute to LPS-induced IL-6 synthesis in mouse peritoneal macrophages. First, consistent with previous observations, we found that LPS induced ROS production in a manner sensitive to DPI or NAC, a free radical scavenger, in peritoneal macrophages (Figure 4a). DPI and NAC also each inhibited LPS-induced IL-6 expression (Figures $4 \mathrm{~b}$ and c), implicating Nox-derived ROS generation in LPS signaling to IL-6 synthesis. To determine which Nox isoforms contribute to
LPS-induced IL-6 production, we examined the effects of LPS on Nox isoforms' expression in peritoneal macrophages. LPS induced the upregulation of Nox1 mRNA (Figure 4d) without affecting the abundance of Nox2 or Nox4 mRNAs (data not shown). Depletion of Nox1 by siRNA transfection resulted in marked inhibition of LPS-induced IL-6 expression (Figures 4d and e). It also reduced the extent of LPS-induced ROS generation (Figure 4f).

On the basis of our previous observations showing that Nox functions downstream of BLT2, ${ }^{17,18,22}$ we hypothesized that LPS might upregulate Nox1 through BLT2 in its signaling to IL-6 synthesis. Consistent with this notion, we observed that LPS-induced Nox1 gene expression and ROS generation were greatly attenuated by siRNA-mediated depletion of BLT2 in both peritoneal macrophages (Figures $4 \mathrm{~g}$ and $\mathrm{h}$ ) and Raw 264.7 cells (data not shown). In addition, we examined the role of MyD88 in LPS-induced Nox1 expression and ROS generation. Transfection with MyD88 siRNA markedly inhibited LPSinduced ROS generation and Nox1 gene expression in both peritoneal macrophages (Figures $4 i$ and $j$ ) and Raw 264.7 cells (data not shown). Conversely, forced expression of MyD88 alone increased the abundance of Nox1 mRNA in peritoneal macrophages (Figure 4k). Together, these results suggested that 
a
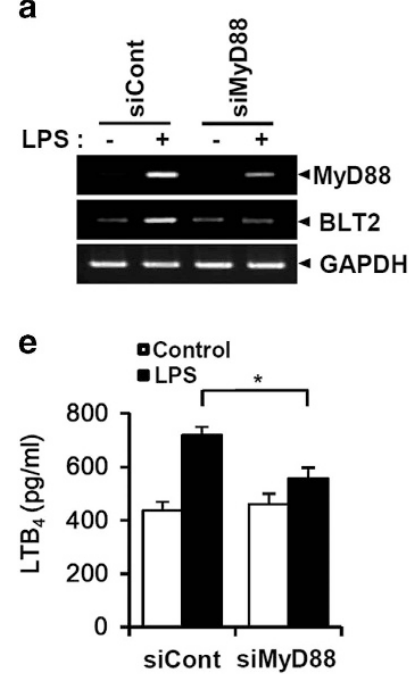

b
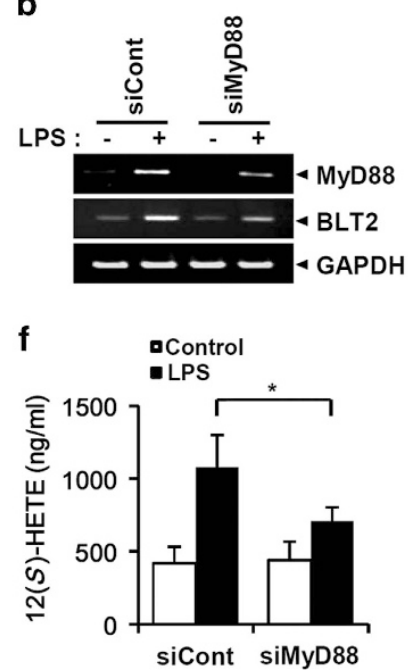

C

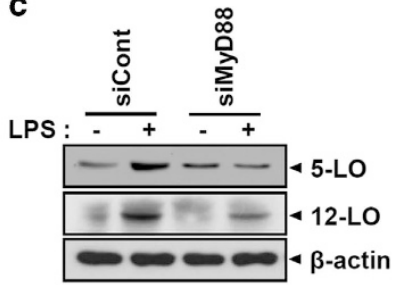

g

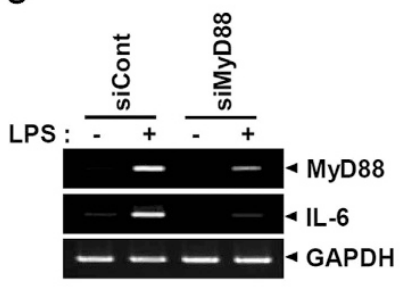

d

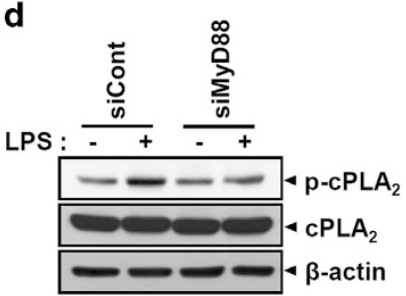

h

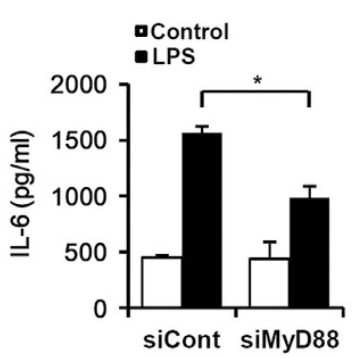

m

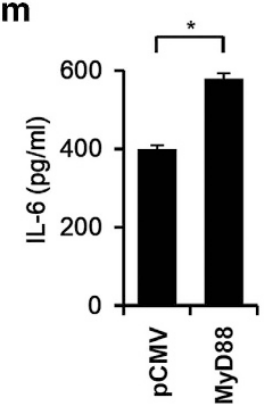

Figure 3 TLR4-MyD88 signaling contributes to LPS-induced BLT2 activation and IL-6 production in mouse macrophages. (a and b) Peritoneal macrophages (a) or Raw 264.7 cells (b) were transfected with control or MyD88 siRNAs, incubated for $24 \mathrm{~h}$ and stimulated with LPS (100 ng ml-1) for $4 \mathrm{~h}$, after which total RNA was isolated and subjected to semiquantitative RT-PCR analysis. (c and d) Raw 264.7 cells were transfected with control or MyD88 siRNAs, incubated for $24 \mathrm{~h}$ and stimulated with LPS for $12 \mathrm{~h}$ (c) or $30 \mathrm{~min}$ (d), after which cell lysates were prepared and subjected to immunoblot analysis. (e-h) Peritoneal macrophages were transfected with control or MyD88 siRNAs, incubated for $24 \mathrm{~h}$ and stimulated with LPS for $12 \mathrm{~h}(\mathbf{e}, \mathbf{f}, \mathbf{h})$ or $4 \mathrm{~h}(\mathbf{g})$, after which LTB 4 (e), 12(S)-HETE (f) or IL-6 (h) released into the culture medium was assayed or total RNA was isolated and subjected to semiquantitative RT-PCR analysis. (i) Raw 264.7 cells were transfected with an expression plasmid for MyD88 and then incubated for $24 \mathrm{~h}$, after which cell lysates were subjected to immunoblot analysis with antibodies to the indicated proteins. (j-m) Peritoneal macrophages (j and $\mathbf{I})$ or Raw 264.7 cells $(\mathbf{k}-\mathbf{m})$ were transfected as in panel (i) and incubated for $24 \mathrm{~h}$, after which $\mathrm{LTB}_{4}(\mathbf{j}), 12(\mathrm{~S})$-HETE (k) or IL-6 (m) released into the culture medium was assayed or total RNA was isolated and subjected to semiquantitative RT-PCR analysis. All quantitative data are means \pm s.d. from three independent experiments. ${ }^{*} P<0.05$.

LPS-MyD88 signaling activates the BLT2-Nox1 cascade to generate ROS that are required for IL-6 synthesis in macrophages.

\section{NF- $\kappa$ B functions downstream of BLT2-Nox1-ROS in LPS-} induced IL-6 synthesis

ROS regulate the activity of NF- $\mathrm{B}$, a transcription factor thought to have a key role in the upregulation of IL- 6 in macrophages. $^{23}$ We also observed that LPS-induced IL-6 expression was greatly inhibited by the NF- $\mathrm{BB}$ inhibitor Bay11-7082 in mouse peritoneal macrophages (Figures 5a and b). In addition, BLT2 depletion resulted in marked attenuation of LPS-induced phosphorylation and degradation of the endogenous NF- $\kappa \mathrm{B}$ inhibitor I $\mathrm{B} \alpha$ (Figure $5 \mathrm{c}$ ). Furthermore, knockdown of Nox1 or treatment with DPI or NAC markedly inhibited LPS-induced NF- $\kappa$ B activation in Raw
264.7 cells (Figures $5 \mathrm{~d}$ and e), suggesting that LPS-induced IL-6 synthesis in mouse macrophages is contributed predominantly through BLT2-Nox1-NF- $\mathrm{B}$ signaling.

\section{DISCUSSION}

LPS is recognized by a variety of cells, including macrophages, and elicits systemic and local host responses. ${ }^{24}$ Stimulation of macrophages with LPS results in the production of various proinflammatory cytokines, such as IL-6 as well as of lipid mediators, such as leukotrienes. ${ }^{24,25}$ IL-6 is an important mediator of the inflammatory response, with IL- 6 levels being increased in individuals with endotoxic shock and sepsis. The mechanism by which LPS induces IL-6 production in macrophages has remained unclear, however, especially with regard to the role of local mediators produced in the endotoxic microenvironment. We have now shown that LPS-induced 
a
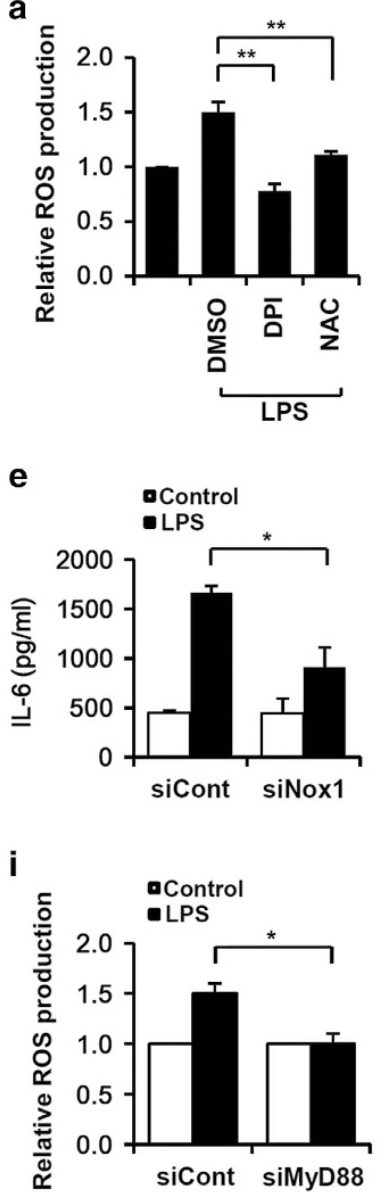

b

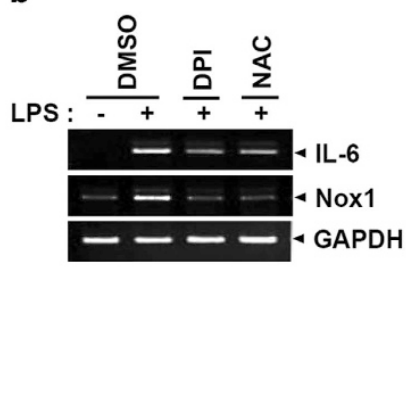

$f$

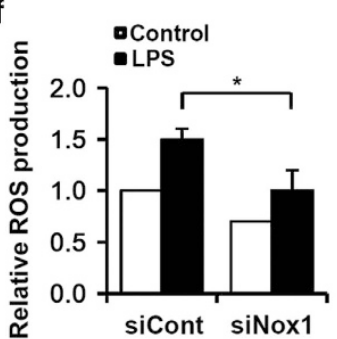

j

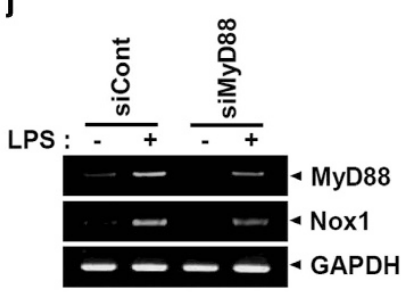

C

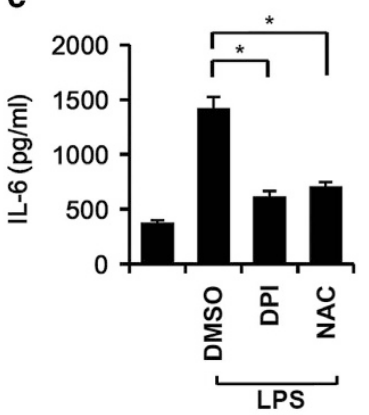

g

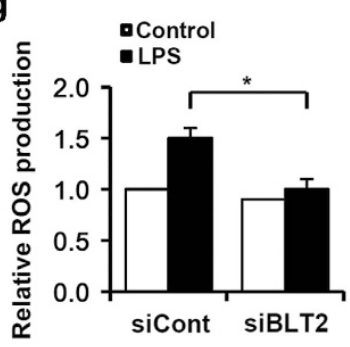

k

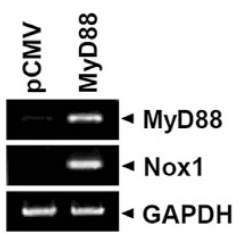

d

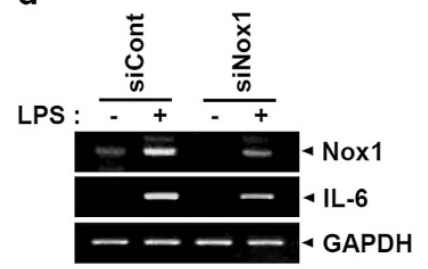

h

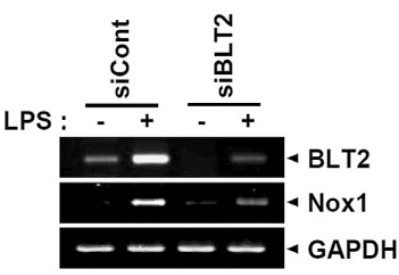

Figure 4 LPS-induced ROS generation and IL-6 synthesis are contributed by BLT2-Nox1 signaling in mouse peritoneal macrophages. (a) Cells were incubated first for 30 min with DPI $(1 \mu \mathrm{m})$, NAC $(1 \mathrm{~mm})$ or DMSO and then for $1 \mathrm{~h}$ in the absence or presence of LPS $\left(100 \mathrm{ng} \mathrm{ml}^{-1}\right) .2^{1}, 7^{\prime}$-Dichlorofluorescein diacetate $(10 \mu \mathrm{m})$ was added for the final $20 \mathrm{~min}$ of the latter incubation, after which intracellular ROS were measured by flow cytometric analysis of DCF fluorescence. (b) Cells were exposed as in panel (a) and then stimulated with LPS for $4 \mathrm{~h}$, after which total RNA was analyzed. (c) Cells were exposed as in panel (a) and then stimulated with LPS for $12 \mathrm{~h}$, after which IL-6 released into the culture medium was assayed. (d) Cells were transfected with Nox1 or control siRNAs, incubated for $24 \mathrm{~h}$ and then stimulated with LPS for $4 \mathrm{~h}$, after which total RNA was analyzed. (e) Cells were transfected and incubated as in panel (d) were stimulated with LPS for $12 \mathrm{~h}$, after which IL-6 released into the culture medium was assayed. (f) Cells were transfected and incubated as in panel (d) were stimulated with LPS for $1 \mathrm{~h}$, after which ROS production was measured. (g and $\mathbf{h}$ ) Cells were transfected with BLT2 or control siRNAs, incubated for $24 \mathrm{~h}$ and then stimulated with LPS for $1 \mathrm{~h}(\mathrm{~g})$ or $4 \mathrm{~h}(\mathrm{~h})$, after which ROS production was measured (g) or total RNA was analyzed (h). (i and j) Cells were transfected with MyD88 or control siRNAs, incubated for $24 \mathrm{~h}$ and stimulated with LPS for $1 \mathrm{~h}$ (i) or $4 \mathrm{~h}$ (j), after which ROS production was measured (i) or total RNA was analyzed (j). (k) Cells were transfected with an expression plasmid for MyD88 or with the empty vector (pCMV) and incubated for $24 \mathrm{~h}$, after which total RNA was analyzed. All quantitative data are means \pm s.d. from three independent experiments. ${ }^{*} P<0.05,{ }^{* *} P<0.01$.

IL-6 synthesis in mouse macrophages is significantly contributed by an $\mathrm{LTB}_{4}-\mathrm{BLT} 2-\mathrm{ROS}-\mathrm{NF}-\mathrm{\kappa B}$ cascade, revealing a link between LPS-MyD88 and the BLT2 signaling pathway.

$\mathrm{LTB}_{4}$ functions as a local pro-inflammatory lipid mediator, acting predominantly in the pathogenic microenvironment. It has a key role in pathological conditions, such as bronchial asthma, rheumatoid arthritis, adult respiratory distress syndrome, as well as endotoxin shock. ${ }^{26-31}$ Leukotrienes trigger or potentiate the synthesis of specific inflammatory cytokines in distinct cell populations, and many cytokines affect cell responsiveness to leukotrienes by modulating the expression levels of their receptors. ${ }^{32}$ Cytokines and bioactive local lipid metabolites such as $\mathrm{LTB}_{4}$ are thus thought to contribute to the pathogenesis of acute and chronic pulmonary inflammation via autocrine or paracrine activation of macrophages. ${ }^{33} \mathrm{LTB}_{4}$ produced in response to platelet-activating factor was previously shown to stimulate IL-6 synthesis in human monocytes. ${ }^{33}$ We have now shown that LPS increases BLT2 expression as well as the production of BLT2 ligands $\left(\mathrm{LTB}_{4}\right.$ and 12(S)-HETE) and that BLT2 knockdown suppressed LPSinduced IL-6 synthesis in mouse macrophages, indicating that BLT2 and its ligands contribute to LPS-induced IL-6 synthesis 
a

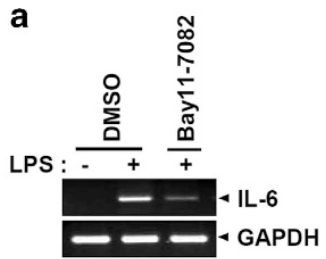

b

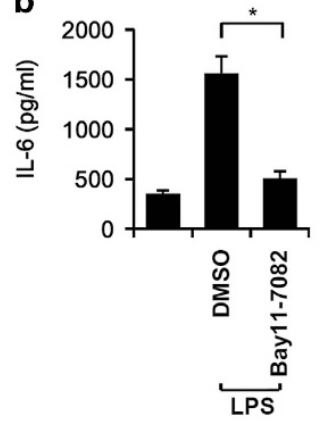

C

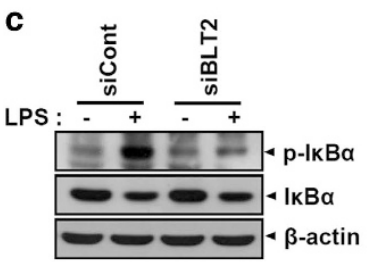

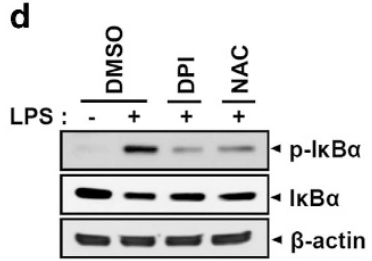

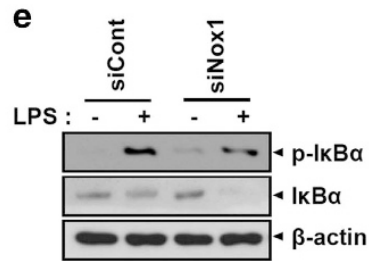

f

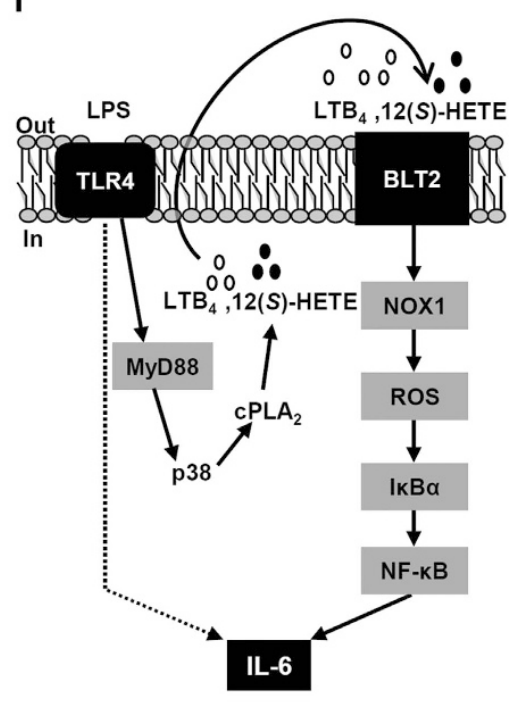

Figure 5 BLT2 contributes to NF-kB activation in LPS-induced signaling to IL-6 synthesis in mouse macrophages. (a and b) Peritoneal macrophages were incubated first for 30 min with Bay11-7082 (10 $\mu \mathrm{m})$ or DMSO and then for $4 \mathrm{~h}(\mathbf{a})$ or $12 \mathrm{~h}$ (b) in the absence or presence of LPS (100 ng ml-1), after which total RNA was isolated and subjected to semiquantitative RT-PCR analysis or IL-6 released into the culture medium was assayed. Data in panel (b) are means \pm s.d. from three independent experiments. ${ }^{*} P<0.05$. (c) Raw 264.7 cells were transfected with BLT2 or control siRNAs, incubated for $24 \mathrm{~h}$ and then stimulated with LPS for 30 min, after which cell lysates were subjected to immunoblot analysis. (d) Raw 264.7 cells were incubated first for 30 min with DPI ( $1 \mu \mathrm{m}$ ), NAC (1 mM) or DMSO and then for $30 \mathrm{~min}$ in the absence or presence of LPS, after which cell lysates were subjected to immunoblot analysis as in panel (c). (e) Raw 264.7 cells were transfected with Nox 1 or control siRNAs, incubated for $24 \mathrm{~h}$ and then stimulated with LPS for 30 min, after which cell lysates were subjected to immunoblot analysis as in panel (c). (f) Scheme for intracellular signaling responsible for LPS-induced IL-6 synthesis in macrophages.

a

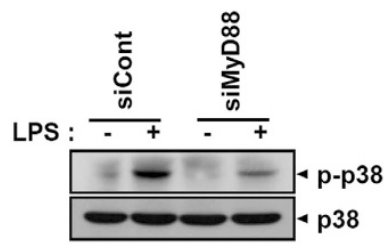

b

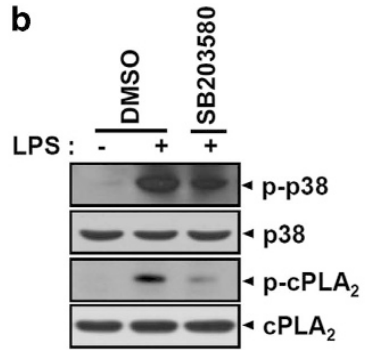

C

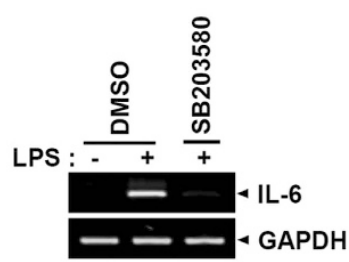

Figure 6 MyD88-dependent activation of p38 MAPK mediates LPS-induced cPLA 2 activation and IL-6 expression in mouse macrophages. (a) Cells were transfected and stimulated with LPS for $30 \mathrm{~min}$. (b and c) Cells were incubated for 30 min with SB203580 (10 $\mu \mathrm{m}$ ) or DMSO and then for 30 min (b) or $4 \mathrm{~h}$ (c) with LPS.

in these cells, most likely through autocrine or paracrine action (Figure 5f). Consistent with the proposed contributing role of BLT2 in the LPS-induced signaling to IL-6 synthesis, BLT2 overexpression alone resulted in a modest increase in IL-6 synthesis, and the addition of BLT2 ligands $\left(\mathrm{LTB}_{4}\right.$ and $12(\mathrm{~S})$ HETE) further enhanced IL-6 synthesis (data not shown).

In the endotoxic microenvironment, MyD88 was first characterized as an essential component of LPS-TLR4 signaling in the activation of innate immunity. ${ }^{34}$ The MyD88-dependent pathway of LPS-TLR4 signaling was found to activate MAPKs, including extracellular signal-regulated kinase, p38 and c-Jun $\mathrm{N}$-terminal kinase, with p38 being thought to be required for LPS-induced activation of CPLA $_{2}$ and lipid release. ${ }^{25}$ MyD88-deficient mice show resistance to LPS-induced responses, including cytokine production by macrophages, and $\mathrm{MyD} 88^{-1-}$ macrophages do not produce IL-6 in response to LPS. ${ }^{35}$ Consistent with these previous observations, we found that transfection of Raw 264.7 cells with MyD88 siRNA inhibited LPS-induced p38 MAPK phosphorylation (Figure 6a) and that the p38-specific inhibitor SB203580 attenuated the LPS-induced phosphorylation of $\mathrm{CPLA}_{2}$ and upregulation of IL-6 mRNA (Figures $6 \mathrm{~b}$ and $\mathrm{c}$ ). We also observed that LPSinduced BLT2 gene expression, $\mathrm{CPLA}_{2}$ activation and the production of BLT2 ligands $\left(\mathrm{LTB}_{4}\right.$ and $12(S)$-HETE) and 
IL-6 were all inhibited by MyD88 knockdown, whereas forced expression of MyD88 alone recapitulated all these effects of LPS. These results suggest that LPS activates a link between MyD88 and BLT2 signaling modules to elicit IL-6 production in macrophages.

Inflammatory stimulants such as LPS induce the generation of ROS, and the induction of ROS in macrophages by LPS is thought to depend on Nox. ${ }^{19,21}$ We have now shown that Nox expression and ROS generation are increased by LPS in peritoneal macrophages. Whereas the basal level of Nox2 was relatively high, that of Nox1 was low and Nox4 was undetectable. However, LPS induced a marked increase in the abundance of Nox1 without affecting that of Nox 2 or Nox4, and transfection of cells with Nox1 siRNA attenuated LPSinduced ROS generation as well as IL-6 production. In addition, we found that LPS-induced upregulation of Noxl mRNA and ROS production were markedly inhibited by knockdown of MyD88 or BLT2. These data thus suggest that Nox1-dependent generation of ROS functions downstream of BLT2 in signaling from LPS to IL-6 synthesis in macrophages. LPS triggers the activation of NF- $\mathrm{\kappa B}$, which has a central role in the regulation of inflammation-related genes such as that for IL-6. ${ }^{36,37}$ We have now shown that LPS-induced IL-6 synthesis was attenuated by the selective NF- $\mathrm{BB}$ inhibitor Bay11-7082 and that activation of NF- $\mathrm{KB}$ was suppressed by the BLT2 knockdown in macrophages. In addition, DPI, NAC and Nox1 siRNA all inhibited LPS-induced NF- $\mathrm{kB}$ activation, suggesting that LPS-BLT2-Nox1 signaling to IL-6 synthesis is contributed by NF- $\mathrm{KB}$.

In the present study, we have shown that MyD88-BLT2Nox1-NF-kB signal contributes to IL-6 production in mouse macrophages. In conclusion, we have defined a previously unsuspected role for BLT2-Nox1 signaling in the stimulation of IL-6 synthesis by LPS in mouse macrophages. LPS upregulates both the BLT2 expression and the production of BLT2 ligands, resulting in enhanced ROS generation through upregulation of Nox1 expression. The accumulation of ROS, in turn, activates NF- $\mathrm{kB}$ and thereby triggers IL-6 gene expression. Our results thus provide a valuable insight into the pathogenesis of endotoxin-induced inflammatory pathogenesis.

\section{CONFLICT OF INTEREST}

The authors declare no conflict of interest.

\section{ACKNOWLEDGEMENTS}

This work was supported by Bio \& Medical Technology Development Program Grant 2012M3A9C5048709, Basic Science Research Program Grant 2012R1A2A2A01044526 through the National Research Foundation funded by the Ministry of Science, Information and Communication Technologies (ICT) and Future Planning, Republic of Korea and the National Research and Development Program for Cancer Control Grant 1220020, Ministry for Health and Welfare, Republic of Korea.
1 Di Rosa R, Pietrosanti M, Luzi G, Salemi S, D'Amelio R. Polyclonal intravenous immunoglobulin: an important additional strategy in sepsis? Eur J Intern Med 2014; 25: 511-516.

2 Greenhill CJ, Rose-John S, Lissilaa R, Ferlin W, Ernst M, Hertzog PJ et al. IL-6 trans-signaling modulates TLR4-dependent inflammatory responses via STAT3. J Immunol 2011; 186: 1199-1208.

3 Song R, Kim J, Yu D, Park C, Park J. Kinetics of IL-6 and TNF-alpha changes in a canine model of sepsis induced by endotoxin. Vet Immunol Immunopathol 2012; 146: 143-149.

4 Rau S, Kohn B, Richter C, Fenske N, Kuchenhoff H, Hartmann K et al. Plasma interleukin-6 response is predictive for severity and mortality in canine systemic inflammatory response syndrome and sepsis. Vet Clin Pathol 2007; 36: 253-260.

5 Palsson-McDermott EM, O'Neill LA. Signal transduction by the lipopolysaccharide receptor, Toll-like receptor-4. Immunology 2004; 113 153-162.

6 Barton GM, Medzhitov R. Toll-like receptor signaling pathways. Science 2003; 300: 1524-1525.

7 Layoun A, Huang H, Calve A, Santos MM. Toll-like receptor signal adaptor protein MyD88 is required for sustained endotoxin-induced acute hypoferremic response in mice. Am J Pathol 2012; 180: 2340-2350.

8 Cronin JG, Turner ML, Goetze L, Bryant CE, Sheldon IM. Toll-like receptor 4 and MYD88-dependent signaling mechanisms of the innate immune system are essential for the response to lipopolysaccharide by epithelial and stromal cells of the bovine endometrium. Biol Reprod 2012; 86: 51.

9 Uozumi N, Kita Y, Shimizu T. Modulation of lipid and protein mediators of inflammation by cytosolic phospholipase A2alpha during experimental sepsis. J Immunol 2008; 181: 3558-3566.

10 Wang X, Xue H, Xu Q, Zhang K, Hao X, Wang L et al. p38 kinase/cytosolic phospholipase A2/cyclooxygenase-2 pathway: a new signaling cascade for lipopolysaccharide-induced interleukin- 1 beta and interleukin- 6 release in differentiated U937 cells. Prostaglandins Other Lipid Mediat 2008; 86: 61-67.

11 Cho KJ, Seo JM, Lee MG, Kim JH. BLT2 Is upregulated in allergen-stimulated mast cells and mediates the synthesis of Th2 cytokines. $\mathrm{J}$ Immunol 2010; 185: 6329-6337.

12 Cho KJ, Seo JM, Shin Y, Yoo MH, Park CS, Lee SH et al. Blockade of airway inflammation and hyperresponsiveness by inhibition of BLT2, a low-affinity leukotriene B4 receptor. Am J Respir Cell Mol Biol 2010; 42: 294-303.

13 Coste I, Le Corf K, Kfoury A, Hmitou I, Druillennec S, Hainaut P et al. Dual function of MyD88 in RAS signaling and inflammation, leading to mouse and human cell transformation. J Clin Invest 2010; 120: 3663-3667.

14 Tager AM, Luster AD. BLT1 and BLT2: the leukotriene B(4) receptors. Prostaglandins Leukot Essent Fatty Acids 2003; 69: 123-134.

15 Powell WS, Gravel S, Khanapure SP, Rokach J. Biological inactivation of 5-oxo-6,8,11,14-eicosatetraenoic acid by human platelets. Blood 1999; 93: 1086-1096.

16 Cho KJ, Seo JM, Kim JH. Bioactive lipoxygenase metabolites stimulation of NADPH oxidases and reactive oxygen species. Mol Cells 2011; 32: 1-5.

17 Choi JA, Lee JW, Kim H, Kim EY, Seo JM, Ko J et al. Pro-survival of estrogen receptor-negative breast cancer cells is regulated by a BLT2-reactive oxygen species-linked signaling pathway. Carcinogenesis 2010; 31: 543-551.

$18 \mathrm{Kim}$ EY, Seo JM, Kim C, Lee JE, Lee KM, Kim JH. BLT2 promotes the invasion and metastasis of aggressive bladder cancer cells through a reactive oxygen species-linked pathway. Free Radic Biol Med 2010; 49: 1072-1081.

19 Kim JS, Yeo S, Shin DG, Bae YS, Lee JJ, Chin BR et al. Glycogen synthase kinase 3beta and beta-catenin pathway is involved in toll-like receptor 4-mediated NADPH oxidase 1 expression in macrophages. FEBS J 2010; 277: 2830-2837.

20 Lee HK, Park DW, Bae JH, Kim HJ, Shin DG, Park JS et al. RGS2 is a negative regulator of STAT3-mediated Nox1 expression. Cell Signal 2012; 24: 803-809

21 Maitra U, Singh N, Gan L, Ringwood L, Li L. IRAK-1 contributes to lipopolysaccharide-induced reactive oxygen species generation in macrophages by inducing NOX-1 transcription and Rac1 activation and suppressing the expression of antioxidative enzymes. J Biol Chem 2009; 284: 35403-35411.

22 Kim GY, Lee JW, Ryu HC, Wei JD, Seong CM, Kim JH. Proinflammatory cytokine IL-1beta stimulates IL-8 synthesis in mast cells via a leukotriene B4 receptor 2-linked pathway, contributing to angiogenesis. J Immunol 2010; 184: 3946-3954. 
23 Andreakos E, Sacre SM, Smith C, Lundberg A, Kiriakidis S, Stonehouse T et al. Distinct pathways of LPS-induced NF-kappa B activation and cytokine production in human myeloid and nonmyeloid cells defined by selective utilization of MyD88 and Mal/TIRAP. Blood 2004; 103: 2229-2237.

24 Shoeb M, Yadav UC, Srivastava SK, Ramana KV. Inhibition of aldose reductase prevents endotoxin-induced inflammation by regulating the arachidonic acid pathway in murine macrophages. Free Radic Biol Med 2011; 51: 1686-1696.

$25 \mathrm{Qi} \mathrm{HY}$, Shelhamer JH. Toll-like receptor 4 signaling regulates cytosolic phospholipase A2 activation and lipid generation in lipopolysaccharide-stimulated macrophages. J Biol Chem 2005; 280: 38969-38975.

26 Fink MP, O'Sullivan BP, Menconi MJ, Wollert PS, Wang $H$, Youssef ME et al. A novel leukotriene B4-receptor antagonist in endotoxin shock: a prospective, controlled trial in a porcine model. Crit Care Med 1993; 21 : 1825-1837.

27 Gelfand EW, Dakhama A. CD8+ T lymphocytes and leukotriene B4: novel interactions in the persistence and progression of asthma. J Allergy Clin Immunol 2006; 117: 577-582.

28 Kim ND, Chou RC, Seung E, Tager AM, Luster AD. A unique requirement for the leukotriene B4 receptor BLT1 for neutrophil recruitment in inflammatory arthritis. J Exp Med 2006; 203: 829-835.

29 Wollert PS, Menconi MJ, O'Sullivan BP, Wang H, Larkin V, Fink MP. LY255283, a novel leukotriene B4 receptor antagonist, limits activation of neutrophils and prevents acute lung injury induced by endotoxin in pigs. Surgery 1993; 114: 191-198.

30 Henderson WR Jr. The role of leukotrienes in inflammation. Ann Intern Med 1994; 121: 684-697.

31 Lewis RA, Austen KF, Soberman RJ. Leukotrienes and other products of the 5-lipoxygenase pathway. Biochemistry and relation to pathobiology in human diseases. N Engl J Med 1990; 323: 645-655.

32 Rola-Pleszczynski M, Stankova J. Cytokine-leukotriene receptor interactions. ScientificWorldJournal 2007; 7: 1348-1358.
33 Thivierge M, Rola-Pleszczynski M. Involvement of both cyclooxygenase and lipoxygenase pathways in platelet-activating factor-induced interleukin-6 production by alveolar macrophages. Ann NY Acad Sci 1994; 725: 213-222.

34 Kawai T, Adachi O, Ogawa T, Takeda K, Akira S. Unresponsiveness of MyD88-deficient mice to endotoxin. Immunity 1999; 11: $115-122$.

35 Sato N, Takahashi N, Suda K, Nakamura M, Yamaki M, Ninomiya T et al. MyD88 but not TRIF is essential for osteoclastogenesis induced by lipopolysaccharide, diacyl lipopeptide, and IL-1alpha. J Exp Med 2004; 200: 601-611.

36 Li DY, Xue MY, Geng ZR, Chen PY. The suppressive effects of Bursopentine (BP5) on oxidative stress and NF-kB activation in lipopolysaccharideactivated murine peritoneal macrophages. Cell Physiol Biochem 2012; 29 : 9-20.

37 Ndengele MM, Muscoli C, Wang ZQ, Doyle TM, Matuschak GM, Salvemini D. Superoxide potentiates NF-kappaB activation and modulates endotoxin-induced cytokine production in alveolar macrophages. Shock 2005; 23: 1861-1893.

(c) (1) (2) This work is licensed under a Creative Commons Attribution-NonCommercial-ShareAlike 3.0 Unported License. The images or other third party material in this article are included in the article's Creative Commons license, unless indicated otherwise in the credit line; if the material is not included under the Creative Commons license, users will need to obtain permission from the license holder to reproduce the material. To view a copy of this license, visit http:// creativecommons.org/licenses/by-nc-sa/3.0/ 